OPEN ACCESS

Edited by:

Christos Volos,

Aristotle University of Thessaloniki,

Greece

Reviewed by:

Shaobo He,

Central South University, China

DaweiDing,

Anhui University, China

*Correspondence:

Chunbiao $\mathrm{Li}$

goontry@126.com

chunbiaolee@nuist.edu.cn

Specialty section:

This article was submitted to Interdisciplinary Physics,

a section of the journal

Frontiers in Physics

Received: 17 August 2021 Accepted: 16 September 2021

Published: 27 October 2021

Citation:

Ma X, Li C, Wang R, Jiang Y and Lei T (2021) Memristive Computation-

Oriented Chaos and

Dynamics Control.

Front. Phys. 9:759913.

doi: 10.3389/fphy.2021.759913

\section{Memristive Computation-Oriented Chaos and Dynamics Control}

\author{
$X \mathrm{Ma}^{1,2}$, Chunbiao $\mathrm{Li}^{1,2 *}$, Ran Wang ${ }^{1,2}$, Yicheng Jiang ${ }^{1,2}$ and Tengfei Lei ${ }^{3}$ \\ ${ }^{1}$ Jiangsu Collaborative Innovation Center of Atmospheric Environment and Equipment Technology (CICAEET) Nanjing University \\ of Information Science and Technology, Nanjing, China, ${ }^{2}$ School of Artificial Intelligence, Nanjing University of Information Science \\ and Technology, Nanjing, China, ${ }^{3}$ Collaborative Innovation Center of Memristive Computing Application (CICMCA), Qilu Institute \\ of Technology, Jinan, China
}

A variable boostable chaotic system and the Hindmarsh-Rose neuron model are applied for observing the dynamics revised by memristive computation. Nonlinearity hidden in a memristor makes a dynamic system prone to be chaos. Inherent dynamics in a dynamic system can be preserved in specific circumstances. Specifically, as an example, offset boosting in the original system is inherited in the derived memristive system, where the average value of the system variable is rescaled linearly by the offset booster. Additional feedback from memristive computation raises chaos, as a case, in the Hindmarsh-Rose neuron model the spiking behavior of membrane potential exhibits chaos with a relatively large parameter region of the memristor.

Keywords: memristive computation, variable boostable chaotic system, hindmarsh-rose neuron model, offset boosting, dynamics control

\section{INTRODUCTION}

Memristor is a new nonlinear component that brings great convivence for chaos generation [1-5] and dynamics control [6-10]. When a memristor is introduced, a dynamical system finds its way to exhibit more complicated evolvements. Whatever in a 3D [11-13] or 4D [14-16] or an even higher dimensional $[17,18]$ system, a memristor does endow abundant dynamics including hidden attractor $[19,20]$, bistability [21,22], tristability [23], extreme multistability [24-27], and megamultistability [28-30]. Memristor, meminductor [31, 32], and memcapacitor $[33,34]$ are in fact representations of special computation, where the current output is critically associated with the past states and the current input. This is essentially a kind of time delay, which brings nonlinear factors and makes the system more prone to be chaos or even hyperchaos [35-37].

In this paper, a memristor-based computation is introduced in a variable boostable chaotic system and the Hindmarsh-Rose neuron model for observing the influence of memory from the memristive computation. As shown in Figure 1, chaos oscillation, and dynamics control are explored and discussed in a variable boostable system and neuron cells. In $A 3 D$ Memristive Chaotic System, the VB14 system is applied to host a memristor, where chaotic oscillation is preserved with the inherent property of offset boosting. The system and component parameter shows their separate power for preserving chaos and revising dynamics . In A Memristive Hindmarsh-Rose Neuron, the Hindmarsh-Rose neuron model is studied as an example to demonstrate the effect of memory. Memristive computation shows its disturbance for chaos production. Some discussion and future work are presented in the last section. 


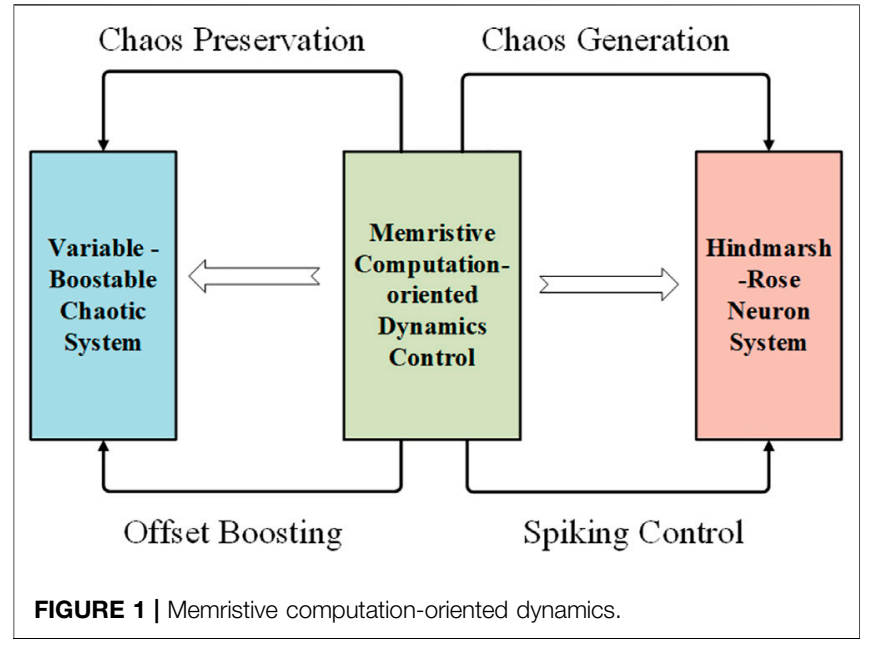

\section{A 3D MEMRISTIVE CHAOTIC SYSTEM}

\section{Basic Analysis}

A variable boostable chaotic system is a class of chaotic systems with an independent variable that can be controlled with a linearly rescaled average value. By introducing a constant, the offset of the variable can be boosted to any desired level. Thus, we can control the variable switch between a bipolar signal and a unipolar signal according to the constant. In this paper, VB14 is selected as the seed system for hosting a memristor,

$$
\left\{\begin{array}{l}
\dot{x}=1-a y z, \\
\dot{y}=z^{2}-z, \\
\dot{z}=x-b z .
\end{array}\right.
$$

when $a=3.55, b=0.5, \mathrm{IC}=(1,0,1)$, system (1) is chaotic with Lyapunov exponents $(0.151,0,-0.651)$ and Kaplan-Yorke dimension $D_{K Y}$ of 2.2319. A memristor is introduced into the VB14 system, as shown in Eq. 2,

$$
\left\{\begin{array}{l}
\dot{x}=1-a(-c+d|y|) z \\
\dot{y}=z^{2}-y z \\
\dot{z}=x-b z
\end{array}\right.
$$

where the flux-controlled memductance is described as,

$$
\left\{\begin{array}{l}
i_{M}=W(y) z \\
W(y)=-c+d|y| \\
\dot{y}=z^{2}-y z
\end{array}\right.
$$

Here $y$ is the internal variable of the memristor and can be regarded as the flux-controlled variable of the memristor. The memristor function curve and the corresponding hysteresis loop are plotted as shown in Figure 2. In the process memristor with the internal variable determined as, $\dot{y}=z^{2}-y z$ is selected for introducing a memristor-based computation. Here $x$, and $z$ are system variables, $a$, and $b$, are the bifurcation parameters of the system (2); and $c$, and $d$ are the internal parameters of the memristor. When $a=2.4, b=0.5, c=1.7$, and $d=2.5$, under initial conditions $(1,0,1)$, system (2) has a chaotic attractor of Lyapunov exponents $(0.18548,0,-1.2907)$ and Kaplan-Yorke dimension of 2.1437, as shown in Figure 3.

The hypervolume contraction of this system can be described by the following expression:

$$
\nabla V=\frac{\partial x}{\partial x}+\frac{\partial y}{\partial y}+\frac{\partial z}{\partial z}=-z-b
$$

which is related to the values of the system variable $z$ and parameter $b$. When system parameters are $a=2.4, b=$ $0.5, c=1.7$, and $d=2.5$, the result of this equation $\nabla V$ is negative. Further examination revealed that the sum of Lyapunov exponents is also negative. So, system (1) is dissipative.

Let the left-hand side of the system (2) be equal to zero, the equilibria can be solved by the following equation:

$$
\left\{\begin{array}{l}
1-a(-c+d|y|) z=0 \\
z^{2}-y z=0 \\
x-b z=0
\end{array}\right.
$$

system (2) has a non-zero equilibrium point $S_{0}=(0.43564387$, $0.87128774,0.87128774)$. Linearizing system (2) at the equilibrium point $\mathrm{S}_{0}$, the Jacobian matrix can be obtained as,

$$
J=\left[\begin{array}{lll}
0 & -a d|y| z & -a(-c+d|y|) \\
0 & -z & -y+2 z \\
1 & 0 & -b
\end{array}\right]
$$

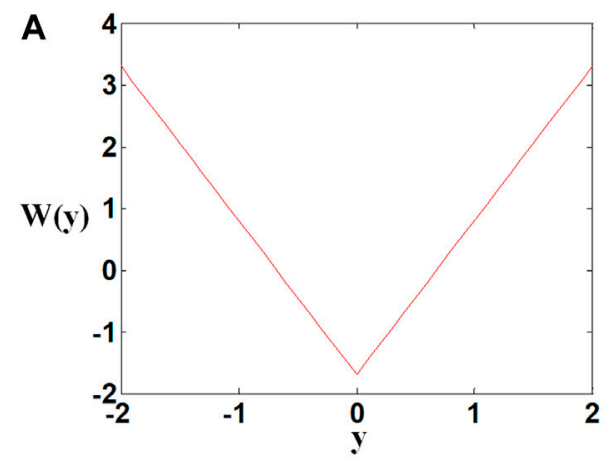

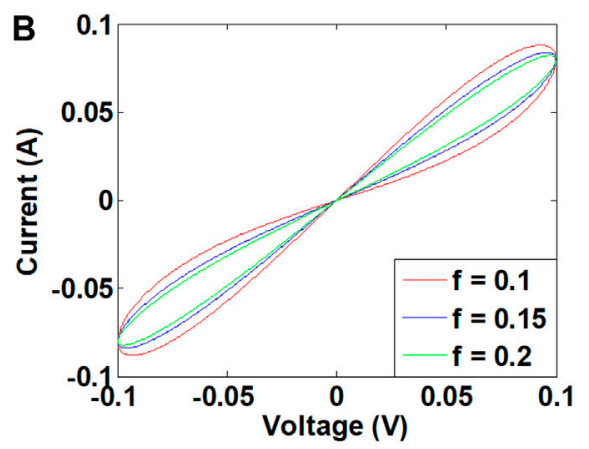

(b)

FIGURE 2 | Memristor function and corresponding hysteresis loop. (A) memristor function, (B) hysteresis loop. 

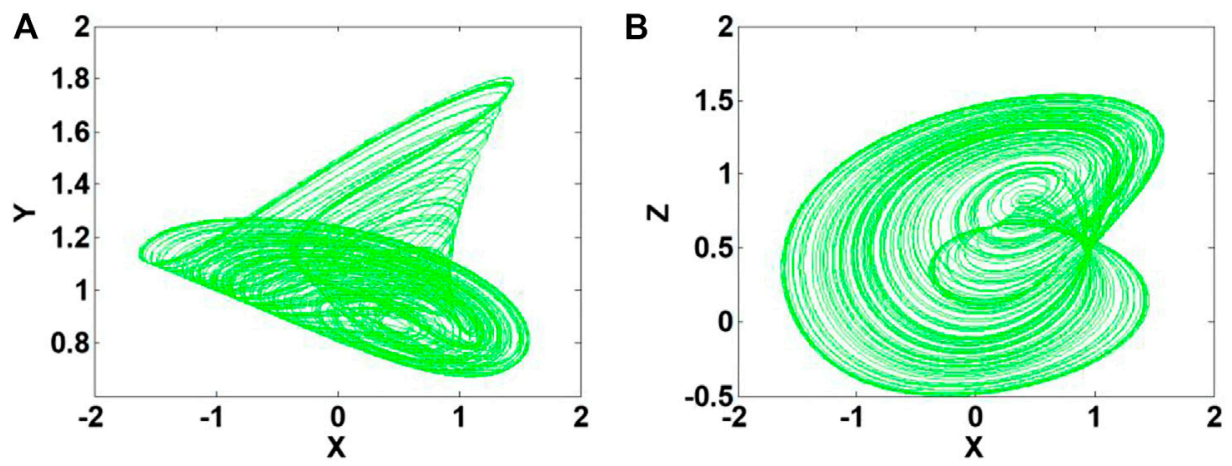

FIGURE 3 | Strange attractor of system (2) with $a=2.4, b=0.5, c=1.7, d=2.5$ under initial conditions $(1,0,1)(\mathbf{A}) x-y$ plane, (B) $x-z$ plane.
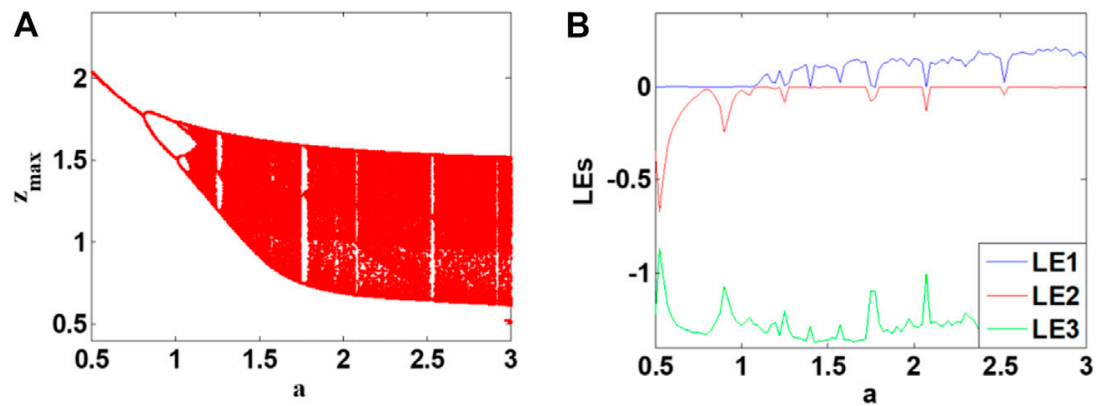

FIGURE 4 | Bifurcation and Lyapunov exponents diagrams of oscillator (2) with $b=0.5, c=1.7, d=2.4$ and the initial conditions is (1, 0,1$)$ when the parameter a is in [0.5, 3]. (A) bifurcation, (B) Lyapunov exponents.
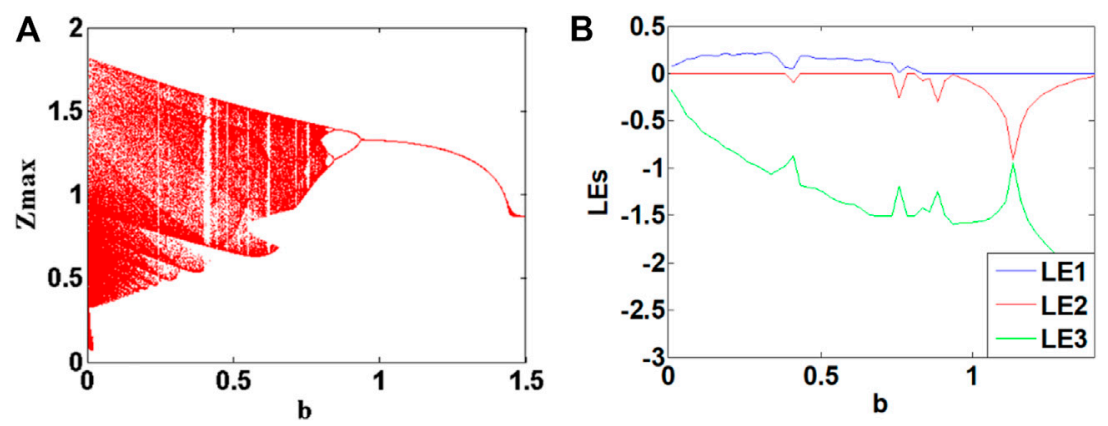

FIGURE 5 | Lyapunov exponents and bifurcation diagrams of system (2) with $a=2.4, c=1.7, d=2.5$ and initial conditions (1, 0 , 1 ) when the parameter $b$ varies in [0, 1.5]. (A) bifurcation, (B) Lyapunov exponents.

The characteristic equation is derived as:

$$
\operatorname{det}(\lambda \mathrm{I}-\mathrm{J})=\left(a_{1} \lambda^{3}+a_{2} \lambda^{2}+a_{3} \lambda+a_{4}\right)=0
$$

Therefore, $\quad a_{1}=1, \quad a_{2}=z+b, \quad a_{3}=a(-c+d|y|)+b z$, $a_{4}=a d|y| z+a(-c+d|y|)$, when $a=2.4, \quad b=0.5, c=1.7$, $d=2.5$, the eigenvalue of $\mathrm{S}_{0}$ is: $\lambda_{1}=-1.9842081$, $\lambda_{2}=0.30646019-1.6448751 i$, and $\lambda_{3}=0.30646019+1.6448751 i$.

\section{Bifurcation Observation}

For system (2), the dynamic behavior of the system can be modified by changing the parameters. When $b=0.5, c=1.7$, $d=2.4$ and the initial condition is $(1,0,1)$, the bifurcation diagram and Lyapunov exponents of parameter $a$ varies in $[0.5,3]$ are shown in Figures $4 \mathbf{A}, \mathbf{B}$. It can be seen from the figure that the system has a couple of periodic windows. When parameter $a$ varies in the range of $[0.5,1.1]$, system (2) is periodic and exhibits a period-doubling process; then, the system enters a chaotic state around $a=1.1$. After that system (2) remains in chaos in [1.1, 1.255], [1.4, 1.725], [1.8, 2.05], [2.1, 2.5], [2.55, 3] except a couple of interrupted period windows.

When $a=2.4, c=1.7, d=2.5$, the initial condition is $(1,0,1)$ and $b$ varies in $(0,1.5)$, the corresponding bifurcation and 

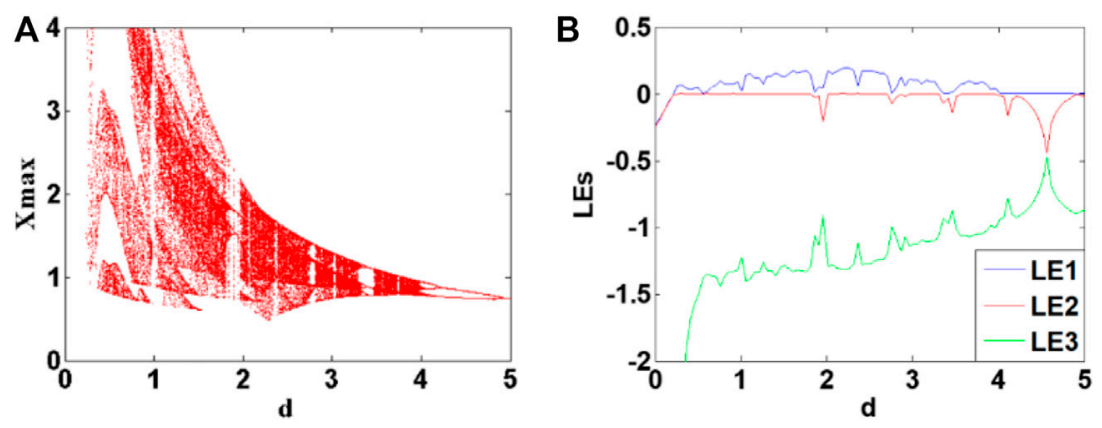

FIGURE 6 | Lyapunov exponents and bifurcation diagrams of system (2) with $a=2.4, b=0.5, c=1.7$ and initial condition (1, 0,1$)$ when the memristor parameter $d$ varies in $[0,5]$. (A) bifurcation, (B) Lyapunov exponents.
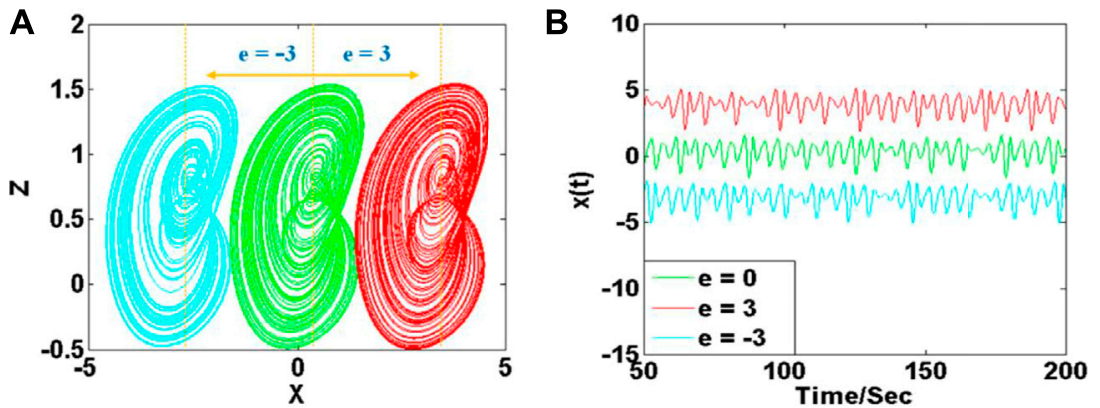

FIGURE 7 | Offset boosted attractors of system (8) with $a=2.4, b=0.5, c=1.7$, and $d=2.5$ : (A) attractors on plane of $x-z$, (B) signal $x(\mathrm{t})$.
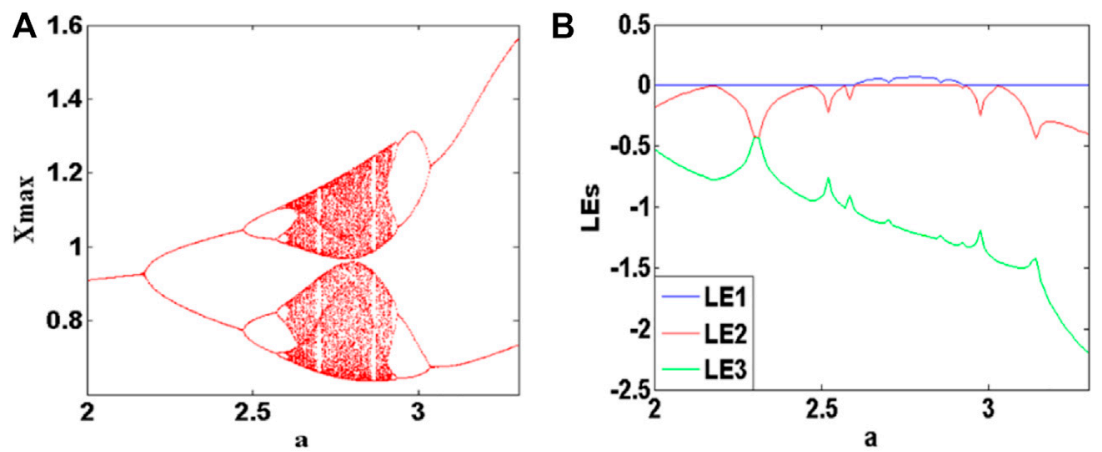

FIGURE 8 Bifurcation and Lyapunov exponents of system (10) with $b=5.5, c=1.7, d=2.5$ and initial condition (0, 0, 0) when parameter a varies in [2, 3.3]. (A) bifurcation, (B) Lyapunov exponents.

Lyapunov exponents are shown in Figure 5. From Lyapunov exponents and bifurcation, we observe that the system undergoes a typical inverse-period-doubling process from chaos. When $b$ in $[0,0.385],[0.435,0.735]$, and $[0.785$, 0.81 ], system (2) exhibits chaos. Some periodic windows such as $[0.385,0.435]$ are seen. When parameter $b$ varies in $[0.81,1.5]$, system (2) gives cycle- 4 , cycle- 2 , and cycle- 1 limit cycles.

To observe the memristive computation influence on system dynamics, here the coefficient $d$ implying memristor resistance is used as a bifurcation parameter. When $a=2.4, b=0.5$, and $c=1.7$, and initial condition $(1,0,1)$, and $d$ increases in the region of $[0,5]$, the bifurcation and Lyapunov exponents are shown in Figures 6A,B. When the internal parameter $d$ of the memristor is in the range of $[1.8,2.01]$, the system shows periodic oscillation; similarly, when the internal parameter $d$ of the memristor is in the range of [2.01, 2.71] and [2.71, $3.56]$, the system again turns to be chaos; when the internal parameter $d$ of the memristor changes in the region of [3.56, 3.96], the system enters a chaotic state. A typical inverse 

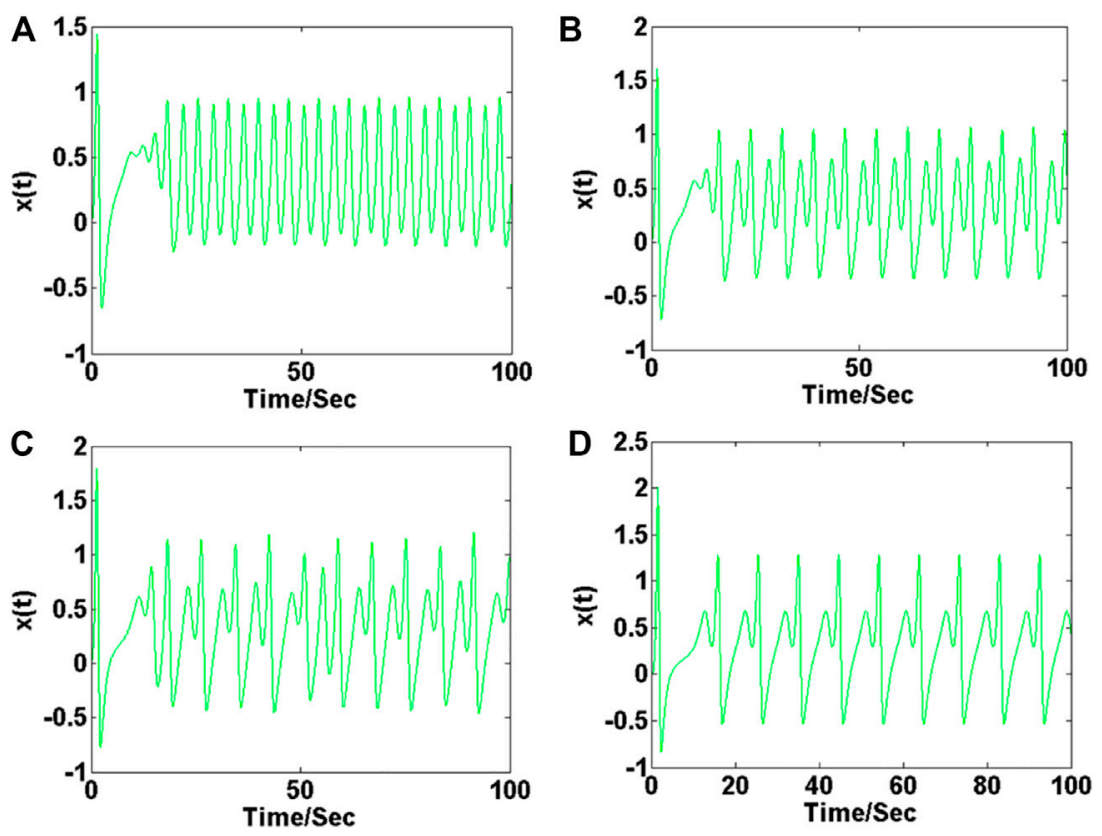

FIGURE 9| Time series of membrane potential under different parameters a of system (10) with $b=5.5, c=1.7, d=2.5$ and initial condition (0, 0, 0): (A) $a=2.2,(\mathbf{B})$ $a=2.5$, (C) $a=2.8$, (D) $a=3$.

periodic doubling bifurcation happens when $d$ becomes larger.

As shown in the latter, the newly introduced memristor does not break the property of offset boosting. Suppose if there is a constant $e$ is introduced in the last dimension,

$$
\left\{\begin{array}{l}
\dot{x}=1-a(-c+d|y|) z \\
\dot{y}=z^{2}-y z \\
\dot{z}=(x-e)-b z
\end{array}\right.
$$

Different constants of $e$ revise the offset of the system variable $x$ directly. As shown in Figure 7, three chaotic attractors with different offsets are displayed in cyan, green, and red. Here signal $x(t)$ at $e=0$ is bipolar signal, while signals at $x(t)$ at $e= \pm 3$ are unipolar positive and negative signal. Offset controller $e$ only rescales the average value of $x$ without influencing system dynamics.

\section{A MEMRISTIVE HINDMARSH-ROSE NEURON}

The 2D HR neuro was simplified from the classical Hodgkin-Huxley model [25] by HR, which is expressed by the following expression,

$$
\left\{\begin{array}{l}
\dot{x}=y-x^{3}+a x^{2}+I \\
\dot{y}=1-b x^{2}-y
\end{array}\right.
$$

where $a$ and $b$ are positive constants, and I is the external current. In the system (9), $x$ stands for membrane potential, and $y$ stands for spiking variable, [38]. To better express the complex dynamical behaviors of the neuron electrical activities, here a new 3-D memristive HR neuron model is proposed [39]. The external current I in the 2-D HR neuron model (8) be replaced by a memristor, the new HR neuron model can be described as,

$$
\left\{\begin{array}{l}
\dot{x}=y-x^{3}+a x^{2}-(-c+d|z|) x, \\
\dot{y}=1-b x^{2}-y, \\
\dot{z}=-x^{2}-x z .
\end{array}\right.
$$

Let the left-hand side of the system (10) be equal to zero, the equilibria can be solved by the following equation:

$$
\left\{\begin{array}{l}
y-x^{3}+a x^{2}-(-c+d|z|) x=0 \\
1-b x^{2}-y=0 \\
-x^{2}-x z=0
\end{array}\right.
$$

System (10) has a non-zero equilibrium point $S=(0.58771263$, - 0.89973376, - and 0.58771263). Linearizing system (10) at the equilibrium point $S_{0}$, the Jacobian matrix can be obtained as,

$$
J=\left[\begin{array}{lrl}
3 x^{2}+2 a x-(-c+d|z|) & 1 & -d x \operatorname{sgn}(z) \\
-2 b x & -1 & 0 \\
-2 x-z & 0 & -x
\end{array}\right]
$$

The characteristic equation is derived as:

$$
\operatorname{det}(\lambda I-J)=\left(a_{1} \lambda^{3}+a_{2} \lambda^{2}+a_{3} \lambda+a_{4}\right)=0
$$

where $a_{1}, a_{2}, a_{3}$, and $a_{4}$ are polynomials containing $a, b, c, d$, and variables $x$ and $z: a_{1}=1, a_{2}=(-3 x+1-2 a) x-c+d|z|+1, a_{3}=$ $-(x+1)\left(3 x^{2}+2 a x-c+d|z|\right)-d x \operatorname{sgn}(z)(2 x+z)+(2 b+1) x$, and $a_{4}=-x\left(3 x^{2}+2 a x+2 b x-d \operatorname{sgn}(z)(2 x+z)-\quad c+d|z|\right)$. When 

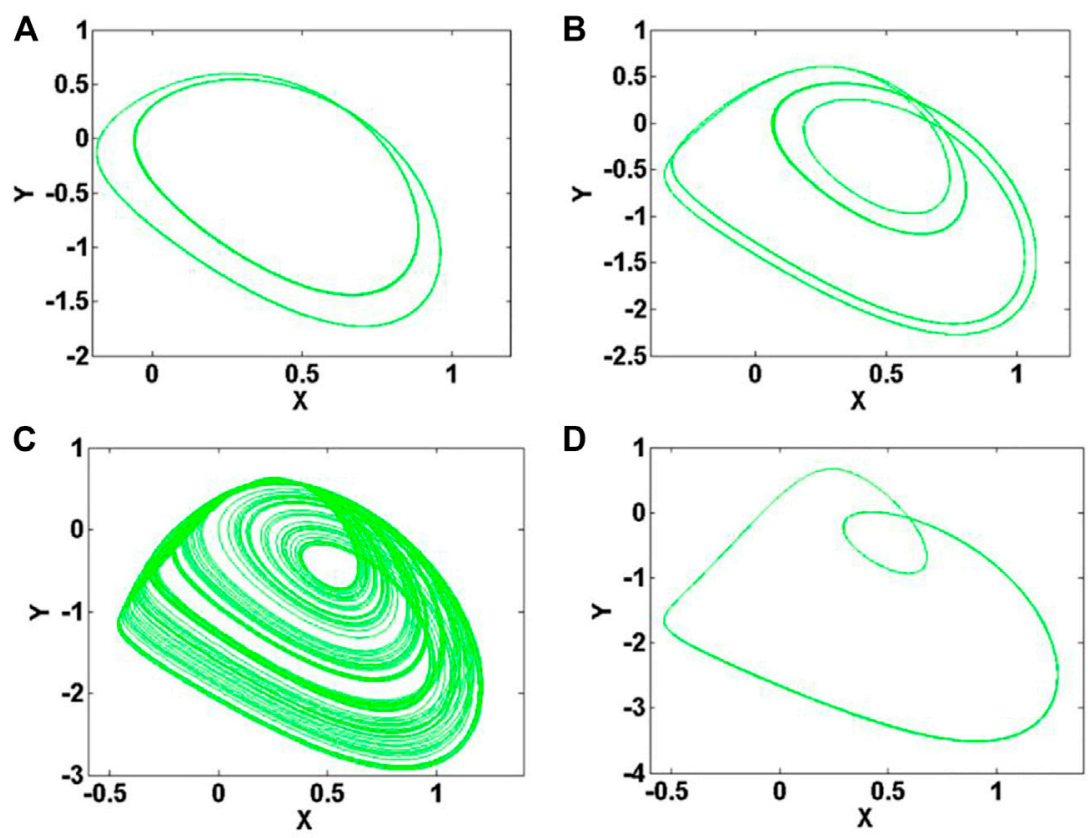

FIGURE 10 | Dynamics of system (10) with $b=5.5, c=1.7, d=2.5$ and initial condition $(0,0,0):(\mathbf{A}) a=2.2,(\mathbf{B}) a=2.5,(\mathbf{C}) a=2.8,(\mathbf{D}) a=3$.
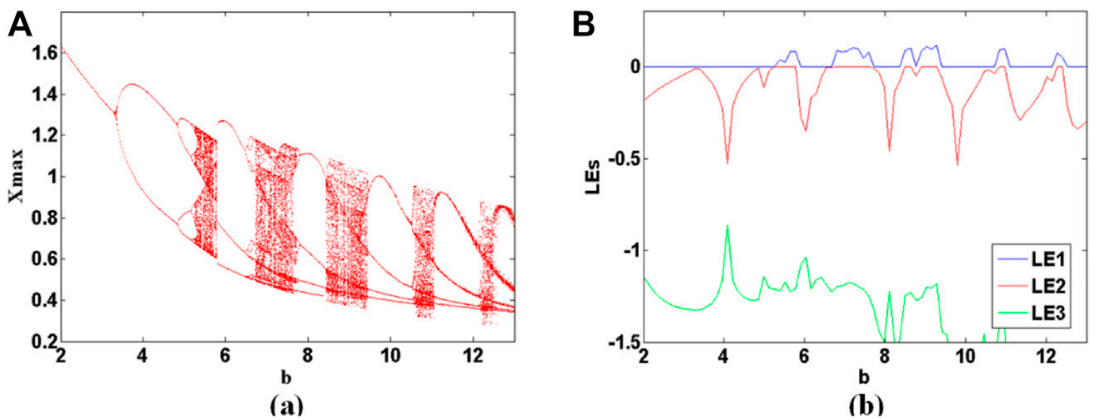

FIGURE 11 Bifurcation and Lyapunov exponents of system (10) with $a=2.8, c=1.7, d=2.5$ and the initial conditions is $(0,0,0)$ when the parameter $b$ varies in $[2$, 13]. (A) bifurcation, (B) Lyapunov exponents.

$a=2.8, b=5.5, c=1.7$, and $d=2.5$, the eigenvalues of $\mathrm{S}$ are: $\lambda_{1}=0.683273$, and $\lambda_{2,3}=0.107353 \pm 0.597675 i$, thus showing it is a saddle point.

The parameters in (10) are specified as follows: $b=5.5, c=$ 1.7 , and $d=2.5$, and the initial condition is $(0,0,0)$. When $a$ varies within the range of $[2,3.3]$, the bifurcation diagram of the membrane potential $x$ and Lyapunov exponents are shown in Figures 8A,B. When the parameter $a$ is increased in the region [2, 2.6], system (10) exhibits typical period-doubling bifurcation; when the range of parameter $a$ varies in $[2.6,2.92]$, system (10) shows chaos; When parameter $a$ is in the range of $[2.92,3.3]$, system (10) exhibits an inverse-period-doubling process. As shown in Figure 9, the neuronal electrical activity shows different modes by modifying the value of the system parameter $a$. Corresponding attractors are displayed in Figure 10.

When $a=2.8, c=1.7$, and $d=2.5$, and the initial condition is $(0,0,0)$, the bifurcation diagram of the maxima $\mathrm{X}$ of the membrane potential $x$ and Lyapunov exponents of parameter $b$ varies in the range of $[2,13]$ are shown in Figures 11A,B. When parameter $b$ is increased in the region of $[0,5.25]$, the system (10) exhibits typical period-doubling bifurcation phenomenon; When parameter $b$ varies in [5.25, 5.77], [6.68, 7.59], [8.5, 8.63], [8.89, 9.28], [10.84, 10.97], and [12.27, 12.4], system (4) exhibits chaos; When parameter $b$ is in the range of $[5.77,6.68],[7.59,8.5],[8.63$ 8.89], [9.28, 10.84], and [10.97, 12.27], system (10) gives periodic solution. When $b$ increases in the region of $[12.27,13]$, system (10) moves from chaos to 

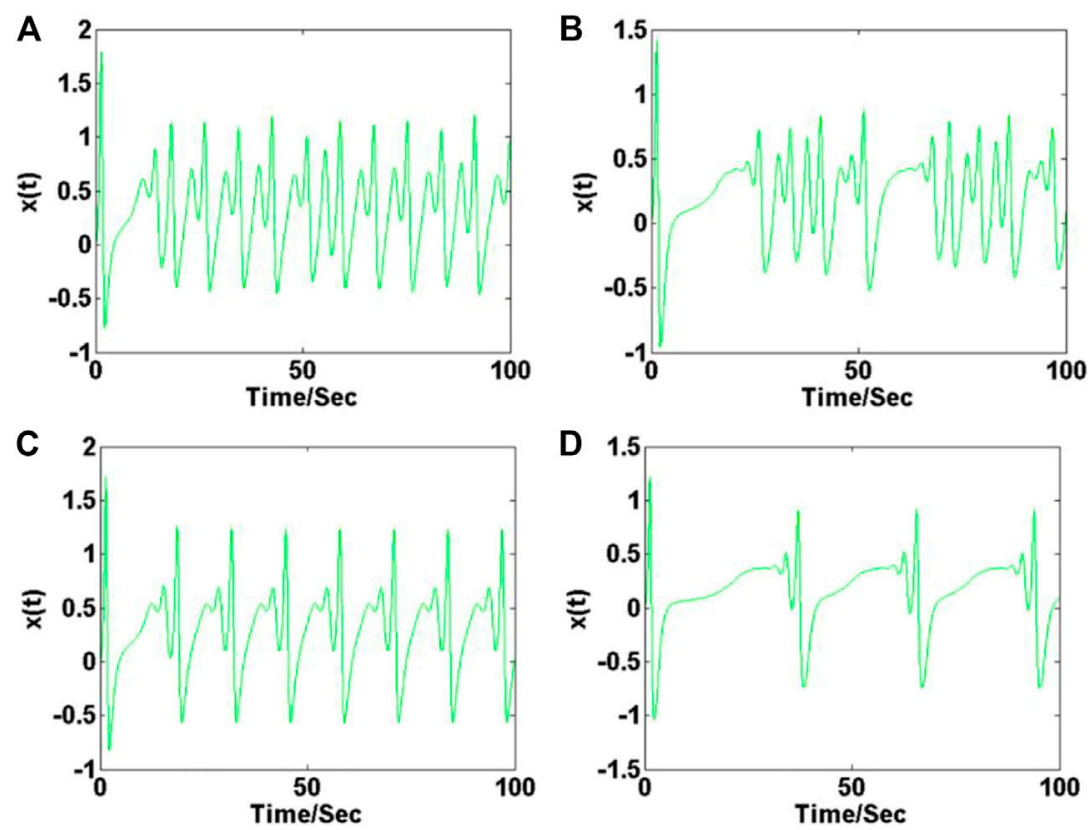

FIGURE 12|Time series of membrane potential of system (10) with $a=2.8, c=1.7, d=2.5$ and initial conditions $(0,0,0):(\mathbf{A}) b=5.5,(\mathbf{B}) b=6.2,(\mathbf{C}) b=9.2,(\mathbf{D})$ $b=11.5$.
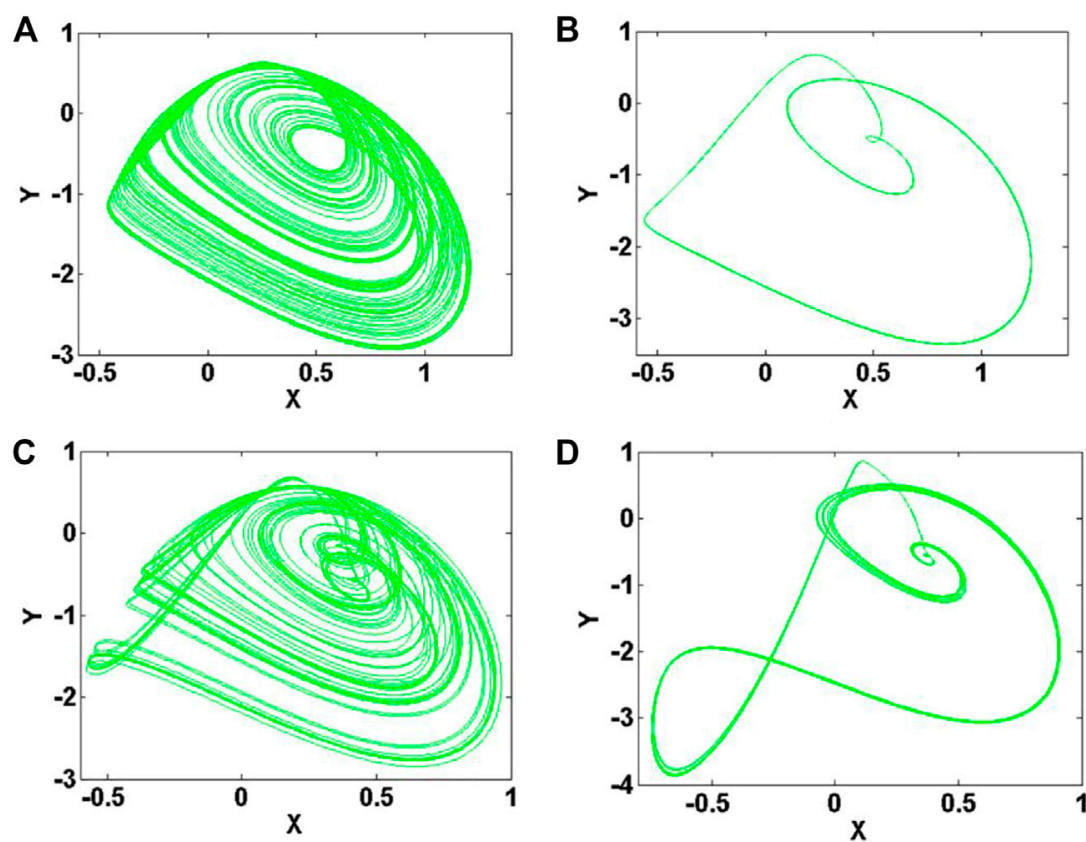

FIGURE 13 | Dynamics of system (10) with $a=2.8, c=1.7, d=2.5$ and initial condition $(0,0,0):(\mathbf{A}) b=5.5$, (B) $b=6.2,(\mathbf{C}) b=9.2,(\mathbf{D}) b=11.5$.

period again. As shown in Figure 12, the bursting state of neuronal electrical activity can be changed into different modes by modifying the value of the system parameter $b$. As shown in Figure 13, attractors correspond to the different bursting states of neuronal electrical activity.
Also to observe the memristive computation influence on system dynamics, here the coefficient $d$ is used as a bifurcation parameter in the derived HR neuron model. When $a=2.4, b=$ $5.5, c=1.7$, and the initial condition is $(0,0,0)$, the bifurcation diagram of the maxima $\mathrm{X}$ of the membrane potential $x$ and 

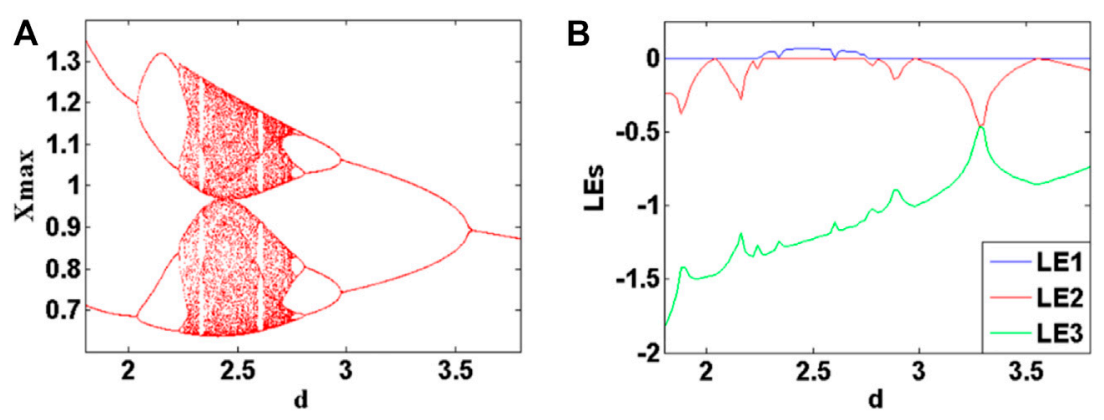

FIGURE 14 | Lyapunov exponents and bifurcation of system (10) with $a=2.8, b=5.5, c=1.7$ and the initial conditions are $(0,0,0)$ when the memristor parameter $d$ varies in $[1.8,3.8]$. (A) bifurcation, (B) Lyapunov exponents.
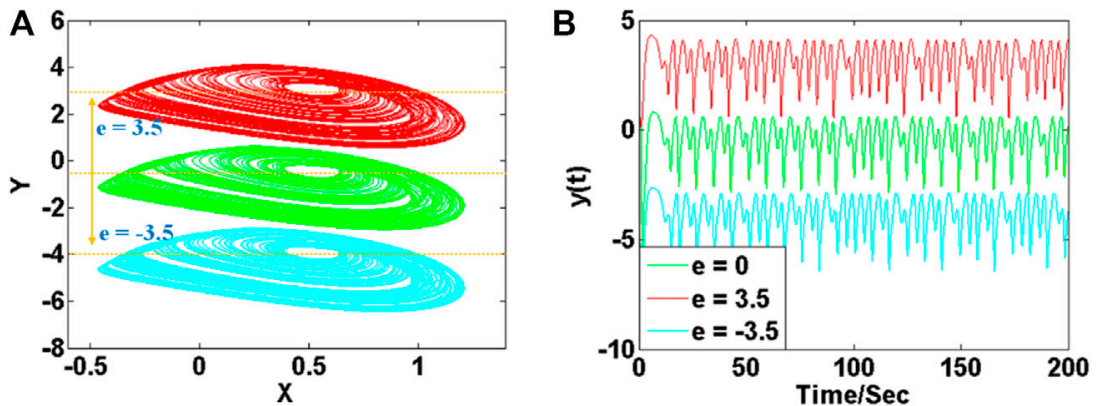

FIGURE 15 | Offset boosted attractors of system (11) with $a=2.8, b=5.5, c=1.7, d=2.5$ : (A) attractors on plane of $x-y$, (B) signal $y(t)$.

Lyapunov exponents of parameter $d$ varies in the range of $[1,4]$ are shown in Figures 14A,B. When the parameter $d$ is increased in the region of $[0,2.24]$, The system exhibits typical period-doubling bifurcation phenomenon; When the range of the parameter $d$ vary in $[2.24,2.75]$, the system (10) is chaotic, obviously, there are two typical period windows in this chaotic range; When parameter $d$ is in the range of $[2.75,4]$, system (10) exhibits an inverse-period-doubling process. Interestingly, the function of the parameter $d$ is similar to the system parameter $a$.

As shown in the latter, the newly introduced memristor does not break the property of offset boosting. Suppose there is a constant $e$ introduced in both of the first dimension and the second dimension,

$$
\left\{\begin{array}{l}
\dot{x}=y-e-x^{3}+a x^{2}-(-c+d|z|) x \\
\dot{y}=1-b x^{2}-(y-e) \\
\dot{z}=-x^{2}-x z
\end{array}\right.
$$

Different constants of $e$ revise the offset of the system variable $y$ directly. As shown in Figure 15, three chaotic attractors with different offsets are displayed in cyan, green, and red. Here signal $x(t)$ at $e=0$ is bipolar signal, while signals at $x(t)$ at $e= \pm 3.5$ are unipolar positive and negative signal. Offset controller $e$ only rescales the average value of $y$ without influencing system dynamics.

\section{CONCLUSION}

Memristor and memristive computation have great merits for producing chaos and dynamics control due to the special nonlinearity. It shows that even a memristor function is a linear function, the memory effect from memristive computation still returns chaos under a specific bifurcation. In this paper, two systems are reformed to be memristive chaotic systems based on the same memristor function. Offset boosting is discussed in both systems. Memristive computation as a new type of computing shows great potential with chaos-based engineering and pattern recognition in artificial intelligence, which deserves further research and will yield great value in information engineering.

\section{DATA AVAILABILITY STATEMENT}

The original contributions presented in the study are included in the article/Supplementary Material, further inquiries can be directed to the corresponding author.

\section{AUTHOR CONTRIBUTIONS}

All authors listed have made a substantial, direct, and intellectual contribution to the work and approved it for publication. 


\section{FUNDING}

This work was supported financially by the National Natural Science Foundation of China (Grant no. 61871230), the Natural

\section{REFERENCES}

Chua L. Memristor-The Missing Circuit Element. IEEE Trans Circuit Theor (1971) 18(5):507-19. doi:10.1109/TCT.1971.1083337

Chua LO, and Sung Mo Kang S. Memristive Devices and Systems. Proc IEEE (1976) 64(2):209-23. doi:10.1109/PROC.1976.10092

Chua L, Komuro M, and Matsumoto T. The Double Scroll Family. IEEE Trans Circuits Syst (1986) 33(11):1072-118. doi:10.1109/TCS.1986.1085869

Chua LO, Komuro M, and Matsumoto T. The Double Scroll Family. Part I: Rigorous Proof of Chaos IEEE Trans (1986) 33(11):1072-1118. doi:10.1109/ TCS.1986.1085869

Deng Q, Wang C, and Yang L. Four-Wing Hidden Attractors with One Stable Equilibrium Point. Int J Bifurcation Chaos (2020) 30(6):2050086. doi:10.1142/ S0218127420500868

Wang R, Li C, Çiçek S, Rajagopal K, and Zhang X. A Memristive Hyperjerk Chaotic System: Amplitude Control, FPGA Design, and Prediction with Artificial Neural Network. Complexity (2021) 2021(1):1-17. doi:10.1155/ $2021 / 6636813$

Lu T, Li C, Wang X, Tao C, and Liu Z. A Memristive Chaotic System with OffsetBoostable Conditional Symmetry. Eur Phys J Spec Top (2020) 229(6):1059-69. doi:10.1140/epjst/e2020-900186-5

Zhang X, Li C, Min F, Iu HH-C, and Gao H. Broken Symmetry in a Memristive Chaotic Oscillator. IEEE Access (2020) 8(8):69222-9. Doidoi:10.1109/ ACCESS.2020.298267110.1109/access.2020.2982671

Gu Z, Li C, Iu HHC, Min F, and Zhao Y. Constructing Hyperchaotic Attractors of Conditional Symmetry. Eur Phys J B (2019) 92(10):221. doi:10.1140/epjb/ e2019-100165-9

Wang C, Xia H, and Zhou L. A Memristive Hyperchaotic Multiscroll Jerk System with Controllable Scroll Numbers. Int J Bifurcation Chaos (2017) 27(6): 1750091. doi:10.1142/S0218127417500912

Li C, Yang Y, Du J, and Chen Z. A Simple Chaotic Circuit with Magnetic FluxControlled Memristor. Eur Phys J Spec Top (2021) 230:1723-36. doi:10.1140/ epjs/s11734-021-00181-2

Yu F, Zhang Z, Shen $\mathrm{H}$, Huang $\mathrm{Y}$, Cai $\mathrm{S}$, Jin $\mathrm{J}$, et al. Design and FPGA Implementation of a Pseudo-random Number Generator Based on a Hopfield Neural Network under Electromagnetic Radiation. Front Phys (2021) 9:690651. doi:10.3389/fphy.2021.690651

Xin B, Peng W, and Guerrini L. A Continuous Time Bertrand Duopoly Game with Fractional Delay and Conformable Derivative: Modeling, Discretization Process, Hopf Bifurcation, and Chaos. Front Phys (2019) 7:00084. doi:10.3389/fphy.2019.00084

Xiu C, Zhou R, and Liu Y. New Chaotic Memristive Cellular Neural Network and its Application in Secure Communication System. Chaos, Solitons \& Fractals (2020) 141:110316. doi:10.1016/j.chaos.2020.110316

Qiao X, Yi X, and Sun Y. Hidden Dynamical Characteristics in Memristor-Based Hyperchaotic Lü System. J Univ Electron Sci Techn China (2018)

He J-J, and Lai B-C. A Novel 4D Chaotic System with Multistability: Dynamical Analysis, Circuit Implementation, Control Design. Mod Phys Lett B (2019) 33(21):1950240. doi:10.1142/S0217984919502403

Ren X, Chen B, Xu Q, Wu H, and Chen M. Parameter and Initial Offset Boosting Dynamics in Two-Memristor-Based Colpitts System. Eur Phys J Spec Top (2021) 230:1709-21. doi:10.1140/epjs/s11734-021-00118-9

Vaidyanathan S, Sambas A, and Azar AT. A 5-D Hyperchaotic Dynamo System with Multistability, its Dynamical Analysis, Active Backstepping Control, and Circuit simulationSystems. Backstepping Control of Nonlinear Dynamical(2021) 449-71. doi:10.1016/B978-0-12-8175828.00025-8

Lin H, Wang C, and Tan Y. Hidden Extreme Multistability with Hyperchaos and Transient Chaos in a Hopfield Neural Network Affected by Electromagnetic
Science Foundation of Jiangsu Province (Grant no. BK20181410), and a Project Funded by the Priority Academic Program Development of Jiangsu Higher Education Institutions.

Radiation. Nonlinear Dyn (2020) 99(4):2369-86. doi:10.1007/s11071-01905408-5

Prousalis DA, Volos CK, Bao B, Meletlidou E, Stouboulos IN, and Kyprianidis IM. Extreme Multistability in a Hyperjerk Memristive System with Hidden Attractors. Chaotic Syst Synchronization (2019) 89-103. doi:10.1016/b978-012-815838-8.00006-6

Li C, Sprott JC, and Xing H. Crisis in Amplitude Control Hides in Multistability. Int J Bifurcation Chaos (2016) 26(14):1650233. doi:10.1142/ S0218127416502333

Li C, Sprott JC, and Thio W. Bistability in a Hyperchaotic System with a Line Equilibrium. J Exp Theor Phys (2014) 118(3):494-500. doi:10.1134/ S1063776114030121

Li Y, Zeng Y, and Zeng J. A Unique Jerk System with Abundant Dynamics: Symmetric or Asymmetric Bistability, Tristability, and Coexisting Bubbles. Braz J Phys (2020) 50(2):153-63. doi:10.1007/s13538-019-00731-z

Yuan F, Deng Y, Li Y, and Wang G. The Amplitude, Frequency and Parameter Space Boosting in a Memristor-Meminductor-Based Circuit. Nonlinear Dyn (2019) 96(1):389-405. doi:10.1007/s11071-019-04795-Z

Bao BC, Bao H, Wang N, Chen M, and Xu Q. Hidden Extreme Multistability in Memristive Hyperchaotic System. Chaos, Solitons \& Fractals (2017) 94:102-11. doi:10.1016/j.chaos.2016.11.016

Zhang S, Zeng Y, Li Z, and Zhou C. Hidden Extreme Multistability, Antimonotonicity and Offset Boosting Control in a Novel Fractional-Order Hyperchaotic System without Equilibrium. Int J Bifurcation Chaos (2018) 28(13):1850167. doi:10.1142/S0218127418501675

Zhang Y, Liu Z, Wu H, Chen S, and Bao B. Extreme Multistability in Memristive Hyper-Jerk System and Stability Mechanism Analysis Using Dimensionality Reduction Model. Eur Phys J Spec Top (2019) 228(10):1995-2009. doi:10.1140/ epjst/e2019-800238-0

Sprott JC, Jafari S, Khalaf AJM, and Kapitaniak T. Megastability: Coexistence of a Countable Infinity of Nested Attractors in a Periodically-Forced Oscillator with Spatially-Periodic Damping. Eur Phys J Spec Top (2017) 226(9):1979-85. doi:10.1140/epjst/e2017-70037-1

Singh JP, Jafari S, Khalaf AJM, Pham V-T, and Roy BK. A Modified Chaotic Oscillator with Megastability and Variable Boosting and its Synchronisation Using Contraction Theory-Based Control Which Is Better Than Backstepping and Nonlinear Active Control. Pramana - J Phys (2020) 94(1):1-14. doi:10.1007/s12043-020-01993-y

Singh JP, Koley J, Lochan K, and Roy BK. Presence of Megastability and Infinitely Many Equilibria in a Periodically and Quasi-Periodically Excited Single-Link Manipulator. Int J Bifurcation Chaos (2021) 31(2):2130005. doi:10.1142/ S0218127421300056

Yuan F, and Li Y. A Chaotic Circuit Constructed by a Memristor, a Memcapacitor and a Meminductor. Chaos (2019) 29(10):101101. doi:10.1063/1.5125673

Zhao Q, Wang C, and Zhang X. A Universal Emulator for Memristor, Memcapacitor, and Meminductor and its Chaotic Circuit. Chaos (2019) 29(1):013141. doi:10.1063/1.5081076

Çam Taşkıran ZG, Sağbaş M, Ayten UE, and Sedef H. A New Universal Mutator Circuit for Memcapacitor and Meminductor Elements. AEU - Int J Elect Commun (2020) 119:153180. doi:10.1016/j.aeue.2020.153180

Akgul A. Chaotic Oscillator Based on Fractional Order Memcapacitor. J Circuit Syst Comp (2019) 28(14):1950239. doi:10.1142/ S0218126619502396

Kong S, Li C, He S, Çiçek SQ, and Lai Q. A Memristive Map with Coexisting Chaos and Hyperchaos. Chin Phys. B (2021). doi:10.1088/1674-1056/ $\mathrm{abf} 4 \mathrm{fb}$

Peng Y, He S, and Sun K. Chaos in the Discrete Memristor-Based System with Fractional-Order Difference. Results Phys (2021) 24:104106. doi:10.1016/ j.rinp.2021.104106 
He S, Sun K, Peng Y, and Wang L. Modeling of Discrete Fracmemristor and its Application. AIP Adv (2020) 10(1):015332. doi:10.1063/1.5134981

Kaslik E. Analysis of Two- and Three-Dimensional Fractional-Order HindmarshRose Type Neuronal Models. Fract Calc Appl Anal (2017) 20(3):623-45. doi:10.1515/fca-2017-0033

Bao $\mathrm{H}, \mathrm{Hu} \mathrm{A}$, Liu W, and Bao B. Hidden Bursting Firings and Bifurcation Mechanisms in Memristive Neuron Model with Threshold Electromagnetic Induction. IEEE Trans Neural Netw Learn Syst. (2020) 31:502-11. doi:10.1109/ TNNLS.2019.2905137

Conflict of Interest: The authors declare that the research was conducted in the absence of any commercial or financial relationships that could be construed as a potential conflict of interest.
Publisher's Note: All claims expressed in this article are solely those of the authors and do not necessarily represent those of their affiliated organizations, or those of the publisher, the editors and the reviewers. Any product that may be evaluated in this article, or claim that may be made by its manufacturer, is not guaranteed or endorsed by the publisher.

Copyright () $2021 \mathrm{Ma}, \mathrm{Li}$, Wang, Jiang and Lei. This is an open-access article distributed under the terms of the Creative Commons Attribution License (CC BY).

The use, distribution or reproduction in other forums is permitted, provided the original author(s) and the copyright owner(s) are credited and that the original publication in this journal is cited, in accordance with accepted academic practice. No use, distribution or reproduction is permitted which does not comply with these terms. 